\title{
MHD stagnation-point flow and heat transfer past a stretching/shrinking sheet in a hybrid nanofluid with induced magnetic field
}

\begin{abstract}
Purpose - The purpose of this paper is to investigate the steady magnetohydrodynamics (MHD) boundary layer stagnation-point flow of an incompressible, viscous and electrically conducting fluid past a stretching/ shrinking sheet with the effect of induced magnetic field. Design/methodology/approach - The governing nonlinear partial differential equations are transformed into a system of nonlinear ordinary differential equations via the similarity transformations before they are solved numerically using the "bvp4c"function in MATLAB. Findings - It is found that there exist non-unique solutions, namely, dual solutions for a certain range of the stretching/shrinking parameters. The results from the stability analysis showed that the first solution (upper branch) is stable and valid physically, while the second solution (lower branch) is unstable. Practical implications - This problem is important in the heat transfer field such as electronic cooling, engine cooling, generator cooling, welding, nuclear system cooling, lubrication, thermal storage, solar heating, cooling and heating in buildings, biomedical, drug reduction, heat pipe, space aircrafts and ships with better efficiency than that of nanofluids applicability. The results obtained are very useful for researchers to determine which solution is physically stable, whereby, mathematically more than one solution exist. Originality/value - The present results are new and original for the problem of MHD stagnation-point flow over a stretching/shrinking sheet in a hybrid nanofluid, with the effect of induced magnetic field.
\end{abstract}

Keyword: Boundary layer; Stability analysis; Hybrid nanofluid; Non-unique solutions; Stagnation-point flow 